\title{
Dilema ético: enfermería y uso de psicofármacos en niños, niñas y adolescentes en el servicio nacional de menores
}

\author{
Ethical dilemma: nursing and use of psychotropic drugs in children and \\ adolescents in the servicio nacional de menores
}

\author{
Dilema Ético: enfermagem e uso de drogas psicotrópicas em crianças e \\ adolescentes no servicio nacional de menores
}

\section{Ricardo Humberto Pérez Abarca ${ }^{1}$ y Jessica Olate Andaur ${ }^{2}$}

\author{
${ }^{1}$ Académico Instructor, Departamento de Enfermería, Universidad de Chile \\ ${ }^{2}$ Académico Asistente, Departamento de Enfermería, Universidad de Chile. Correo electrónico: \\ jolate@med.uchile.cl \\ Cómo citar este artículo en edición digital: Pérez Abarca, R.H. \& Olate Andaur, J. (2018). Dilema ético: \\ enfermería y uso de psicofármacos en niños, niñas y adolescentes en el servicio nacional de menores. \\ Cultura de los Cuidados (Edición digital), 22(51). Recuperado de \\ <http://dx.doi.org/10.14198/cuid.2018.51.12> \\ Correspondencia: Departamento de Enfermería, Facultad de Medicina, Universidad de Chile, Av. \\ Independencia 1027, Santiago, Chile, código postal: 8330015 \\ Correo electrónico: ricardo.perez@uchile.cl \\ Recibido: 23/09/2017; Aceptado: 14/02/2018.
}

\begin{abstract}
Objective: Reflect about Nursing and the use of psychoactive drugs in children and adolescents in situations of violation of rights and / or criminal situation, who are in the care of the Servicio Nacional de Menores, in the context of deaths in recent years. Methodology: Analytical-synthetic method; Bibliographical review, comprehensive reading, personal reflection and definition of concepts. Results: There are described the current situation of mental health of children and adolescents, the relationship with the Social Determinants of Health and related ethical perspectives for conflict resolution. Conclusions: The use of psychosocial interventions as the first line of treatment is considered in the reflection, besides the fact that the use of psychoactive drugs, if necessary, should be administered and
\end{abstract}

supervised by professionals according to current regulations.

Keywords: Nursing, children, adolescent, psychoactive drugs, vulnerability.

\section{RESUMEN}

Objetivo: Reflexionar sobre el rol de la enfermería y el uso de psicofármacos en niños, niñas y adolescentes en situación de vulneración de derechos y/o situación penal, que están al cuidado del Servicio Nacional de Menores, en el contexto de las muertes ocurridas en los últimos años. Metodología: Método analítico-sintético; revisión bibliográfica, lectura comprensiva, reflexión personal y definición de conceptos. Resultados: Se describe la situación de salud mental actual infanto-juvenil, la relación con los Determinantes Sociales de Salud y perspectivas 
éticas relacionadas para resolución de conflictos. Conclusiones: Se plantea en la reflexión el uso de las intervenciones psicosociales como primera línea de tratamiento, además de, que el uso de psicofármacos, en caso de ser necesario, debe ser administrado y supervisado por los profesionales conforme a lo que dicta la normativa vigente.

Palabras Clave: Enfermería, niños, adolescentes, psicofármacos, vulnerabilidad

\section{RESUMO}

Objetivo: Refletir sobre Enfermagem e uso de drogas psicotrópicas em crianças e adolescentes em situações de violações de direitos e / ou situação criminosa sob os cuidados do Serviço Nacional de Menores, no contexto de mortes nos últimos anos. Metodologia: metodolo- sintético; revisão da literatura, compreensão de leitura, reflexão pessoal e definição de conceitos. Resultados: situação da saúde mental da criança atual e adolescentes descreve o relacionamento com os Determinantes Sociais da Saúde e perspectivas éticas relacionadas com a resolução de conflitos. Conclusões: O uso de intervenções psicossociais como tratamento de primeira linha, e surge na reflexão, que o uso de drogas psicotrópicas, se necessário, deve ser administrada e supervisionada por profissionais de acordo com os ditames da legislação vigente

Palavras-chave: Enfermagem, crianças, adolescentes, psicotrópicos, a vulnerabilidade

\section{INTRODUCCIÓN}

La problemática de la Psiquiatría y Salud Mental durante la historia ha sido un tema controversial. Vale la pena referir, por ejemplo; la lobotomía y la terapia electroconvulsiva, como parte, incluso, de un pasado reciente. Aunque todavía más; la inclusión de los psicofármacos, genética, y sistemas computarizados de monitoreo de las funciones neuroquímicas que se encuentran en desarrollo. A lo anterior, las discusiones reflejadas en la quinta edición del Manual Diagnóstico y Estadístico de los Trastornos Mentales (DSM V), elaborada y publicada por la Asociación Americana de Psiquiatría en el 2014, explicita la dificultad ética y técnica de diagnosticar e intervenir la mente humana. Se podría inferir, que lo mencionado no está resuelto, y el debate es más actual que nunca.

La intención de esta reflexión no es abrir estrictamente la discusión sobre la Psiquiatría y Salud Mental (diagnósticos y tratamientos, menos su historia); ni tampoco ahondar en la profundidad filosófica sobre Libertad y Autonomía; ni siquiera mencionar Estado y Responsabilidad (en el marco judicial en que Chile adscribe actualmente basado en principios fundamentales de la humanidad, fuertemente desarrolladas por instituciones internacionales como la Organización Mundial de la Salud[OMS]); sino plantear que; las características y condiciones institucionales en que se encuentran niños, niñas y adolescentes (NNA) dependientes del Servicio Nacional de Menores (SENAME), que presentan problemas de salud mental, junto con el antecedente de 1.313 muertes que se han revelado durante el mes de junio 2017, permitiría visualizar como primera línea de abordaje las intervenciones psicosociales especializadas, por ser más pertinentes que las psicofarmacológicas. Las cuales, en caso de requerirse, deben ser indicadas y supervisadas por profesionales de salud, que garanticen la administración segura, junto con la detección y manejo precoz de complicaciones.

\section{METODOLOGÍA}

Se utilizó el método analítico-sintético; revisión bibliográfica, lectura comprensiva, reflexión personal y definición de conceptos. La búsqueda incluyó las palabras: Servicio Nacional de Menores, enfermería, biopoder, ética, salud mental, psicofármacos. Fue realizada en distintas bases de datos nacionales e internacionales.

Criterios de Inclusión: Artículos en español e inglés en base de datos CINAHL, Scopus, Scielo. Publicados entre los años 2007 2017, full text.

Criterios de exclusión: Artículos en idiomas distintos a español o inglés, full text no disponible.

\section{DESARROLLO DEL TEMA $Y$ SU DISCUSIÓN}

En primer lugar, es necesario ahondar sobre la influencia del marketing de las farmacéuticas en 
médicos y la salud mental de consumidores, y si es éticamente correcto la prescripción de neurofármacos de uso prolongado de manera exclusiva 0 por sobre los tratamientos psicosociales especializados.

La Organización Mundial de la Salud (OMS), en el Plan de Acción en Salud Mental 20132020, refiere la recuperación como uno de los objetivos de la atención, sin embargo, las investigaciones de ensayos clínicos pueden dar cuenta de ésta como resultado, precisamente porque “recuperación” no sólo es difícil de medir o cuantificar sino también de definir. Además, se ha desarrollado a lo largo de la historia del uso de medicamentos en los trastornos mentales, la discusión acerca de que ellos sólo atenúan los síntomas, pero no "curan” la enfermedad, siendo la esquizofrenia un ejemplo de ellos; los antipsicóticos, son efectivos en la sintomatología positiva, sin embargo, la baja adherencia de causa multifactorial conlleva a tratamientos poco efectivos (Saldivia, 2016). Otros autores van más allá, y refutan las intervenciones farmacológicas, pues están basados sólo en un principio positivista, que cuantifica “desregularizaciones fisiológicas” (Whitaker L., 2015).

Existen múltiples factores que influyen en la problemática planteada, y que no necesariamente son de la esfera única de la salud como fenómeno. Se podría mencionar la influencia que tienen los medios de comunicación masivos, la legislación vigente, el modo de vida, etc. Precisamente en la mitad del siglo pasado, movimientos iniciados en distintas partes del mundo, abren la discusión masiva sobre la justicia, igualdad, libertad y ser- En cuyo contexto; surgen las intervenciones psicosociales como respuestas en distintos aspectos de la salud mental de las personas. Centrado en la acción comunitaria, fomento en toma de decisiones y desarrollo de habilidades y capacidades (Pérez C., 2015).

Actualmente, es posible establecer que las intervenciones psicosociales, como el empleo con supervisión y la psicoeducación familiar de medicamentos, reducen las recaídas y pueden ayudar a los pacientes a lograr una vida en la comunidad. Lamentablemente, según Saldivia (2016) aún no se sabe cuántos o cuáles de los pacientes con esquizofrenia se recuperarán con el uso combinado de las intervenciones médicas y psicosociales, debido a la dificultad que tiene la medición y la conversión a resultados de las investigaciones.

La OMS ha establecido que los trastornos psiquiátricos que se inician en la infancia o adolescencia deben ser una prioridad para la Salud Pública, considerando como fundamental detectar y tratar a tiempo, pues esto determina el curso de vida de los niños (OMS, 2013). Lo anterior, se basa en estudios retrospectivos, que demuestran que la edad de inicio de un número importante de trastornos ocurre antes de lo que se estimaba (Burke, 1990) y que hasta un 50\% de los trastornos mentales de los adultos comienzan antes de los catorce años. (Organización Mundial de la Salud, 2013).

Cabe destacar que la OMS, además, declara en su plan 2013-2020: “. Los niños y adolescentes con trastornos mentales deben ser objeto de intervenciones tempranas científicamente contrastadas de carácter no farmacológico, ya sean psicosociales o de otra índole, dispensadas desde el ámbito comunitario, evitando la institucionalización y la medicalización. Esas intervenciones deben atenerse además a los derechos que amparan a los niños con arreglo a la Convención de las Naciones Unidas sobre los Derechos del Niño y otros instrumentos internacionales y regionales de derechos humanos".

Según la OMS, Chile se encuentra entre los países con mayor carga de morbilidad por enfermedades psiquiátricas $(23,2 \%)$ en el mundo. La depresión mayor y los trastornos por consumo de alcohol ocupan el primer y segundo lugar en las discapacidades atribuidas entre adultos. Casi un tercio de la población mayor de 15 años ha sufrido un trastorno psiquiátrico en su lapso de vida y un 22,2\% ha tenido uno durante el año pasado. Solo el 38,5\% de quienes han sido diagnosticados reciben algún tipo de servicio de salud mental, ya sea de un especialista o un médico de atención primaria (Saldivia, 2016).

En niños y adolescentes, la prevalencia de cualquier trastorno psiquiátrico es de 22,5\% (19,3\% para niños y 25,8\% para niñas). Estos trastornos son principalmente de ansiedad $y$ disruptivos, sumado a los de la esfera del ánimo. Los resultados demuestran que más de un tercio 
de la población infanto-juvenil en Chile presenta algún trastorno psiquiátrico en un período de 12 meses. Marmot \& Bell (2012) plantean una nueva visión de la salud basada en la promoción, y que fue desarrollada en los años 60 y 70 en Canadá, siendo sustantivo considerar determinantes sociales que afectan a ella, y que relaciona las condiciones en que las personas, nacen, crecen, viven y trabajan, y que reducir las inequidades podría mejorar la calidad de vida de las personas. En relación a ello, en Chile, el nivel socioeconómico más alto, es el único que aparece asociado significativamente a la menor presencia de trastornos ansiosos, otros estudios han encontrado asociaciones más fuertes con trastornos externalizantes que internalizantes (Vicente et al., 2012). Esto pudiera ser evidencia de la influencia de los Determinantes Sociales de Salud en la salud mental de la población infantojuvenil.

Sumando a lo antes mencionado, relación a las inequidades existentes, Chile tiene 4,0 psiquiatras por 100.000 habitantes. En promedio, esta tasa es semejante a la de países latinoamericanos pero muy por debajo que la de la mayoría de los países desarrollados. En contraste, tendríamos 15,7\% psicólogos por cada 100.000 habitantes. El cuidado de la salud mental y los especialistas para niños y adultos mayores son limitados y, en la mayoría de los casos, no están disponibles. Adicionalmente, existe una amplia disparidad de los recursos disponibles a través del país, y la concentración de especialistas en el mundo privado ha sido una tendencia exponencial en las últimas décadas, como también en las grandes ciudades (Vicente et al., 2012). En Chile, el número de enfermeras es también relativamente bajo, con 4.2 enfermeras por cada 1,000 habitantes en el 2012, comparado con un promedio de 8.8 en los países de la Organización para la Cooperación y Desarrollo Económico (OCDE). Esto se suma a la baja inversión en salud, llegando a un 7.3\% del Producto Interno Bruto; el 49\% del gasto en salud fue financiado por fuentes públicas en 2012, una proporción muy por debajo del promedio de $72 \%$ de los países contrastados $\mathrm{y}$, casi una tercera parte del gasto en salud en Chile es pagado directamente por los hogares, comparado con menos de un $20 \%$ en promedio (OCDE, 2014).
A pesar de los avances en materia de protección de la salud infanto-juvenil, como los Programas de Chile Crece Contigo y el Programa de Salud del Adolescente y el Plan de Salud Mental, los resultados en materia de salud mental no han tenido los efectos esperados. En efecto, por ejemplo, en Chile, los suicidios representan el $60 \%$ del total de las muertes en adolescentes. En el grupo entre 10 a 14 años, la tasa aumentó de 1,0 por 100.000 el año 2000 a 1,9 por 100.000 el año 2009. Del mismo modo, en el grupo de 15 a 19 años, aumentó de 8,6 a 11,4 por 100.000 en el mismo periodo (Departamento de estadísticas e información en salud [DEIS], 2010).

En los últimos 17 años se ha realizado un esfuerzo considerable para desarrollar técnicas de detección temprana basados en prevención y promoción de la salud mental. El desarrollo de todas las carencias infantiles tiene relación con varias variables, como las habilidades que madres y padres poseen para la crianza, la posibilidad de contar con acceso a jardín infantil, las estrategias de prevención de enfermedades y promoción de la salud, condiciones medioambientales, aspectos socioeconómicos y culturales en las que se crece, etc. (Ministerio de Salud [MINSAL], 2012).

En relación a lo anterior, existe un grupo de la población, que se encuentra más expuesto a los trastornos de salud mental, en parte, debido a que están en una situación de vulneración de derechos o han infringido la ley, de manera que, a través de los Tribunales de Justicia son derivados al Servicio Nacional de Menores.

La misión del Servicio Nacional de Menores (SENAME) es contribuir a la promoción, protección y restitución de derechos de niños, niñas y adolescentes vulnerados/as, así como a la responsabilización y reinserción social de los adolescentes infractores/as de leyrecordar que en Chile la responsabilidad penal es a partir de los 14 años-, a través de programas ejecutados directamente o por organismos colaboradores del servicio (SENAME, 2016).

Según el boletín estadístico anual del 2015 del SENAME, son 1623 programas asociados a la atención de niños, niñas y adolescentes (NNA). En ellos, se atiende una población estimada en 106760 personas. Según el mismo informe, son 
376 organismos colaboradores y sólo 62 centros son administrados directamente por la organización estatal. Se estima en 4000 trabajadores, de los cuales el 70\% establecen intervenciones directas con NNA. El mismo boletín establece que las causas más frecuentes asociadas a los ingresos de las personas vigentes en los programas se cuenta en un $44 \%$ por víctima de maltrato, seguido por la inhabilidad de uno o ambos padres con un 15\% de los casos, $\mathrm{y}$, en tercer motivo, el abuso sexual con un $10 \%$ de los casos, seguido por un 9\% como medida de protección resuelta por algún tribunal. Según los mismos datos, alrededor de 13000 niños, niñas y adolescentes presentan algún problema de consumo de drogas. Por su parte, alrededor de dos mil niños presentan algún tipo de problema de salud mental severo diagnosticado.

En el año 2010 la Comisión Económica para América Latina y el Caribe (CEPAL) alertaba sobre lo que implica el sistema de colaboración entre el Estado y la Sociedad Civil (entendido aportes privados), y que las principales falencias estarían por un inadecuado diálogo entre ambas partes, y sugiere que sea el Estado quien debería dictar los lineamientos que debe tomarse en materias de protección infantil. Resalta que no existe legislación en estas materias, y cuestiona la forma de financiamiento y adjudicación de los proyectos vinculados (CEPAL, 2010).

El convenio de colaboración establecido entre el MINSAL y SENAME está orientado a garantizar el acceso oportuno de los niños, niñas y adolescentes a las prestaciones de salud primarias y a las de mayor complejidad, incluyendo episodios de urgencia psiquiátrica. Además, según declara la institución, se dispone de una agenda de trabajo planificada por ambas instituciones mencionadas, $y$, el Servicio Nacional para la Prevención y Rehabilitación del Consumo de Drogas y Alcohol (SENDA), con la finalidad de cautelar la implementación del modelo de atención integral que aborda necesidades en salud mental de los adolescentes (SENAME, 2016). A pesar de ello, no se ha encontrado información sobre dicha implementación de evaluación sobre las necesidades de cada NNA, siendo relevante dada la situación en que se encuentran, un enfoque holístico e interdisciplinar.
Particularmente en estos centros de internación continua se desarrolla un conflicto ético del manejo de los problemas de salud mental mediante el uso de psicofármacos del uso de los psicofármacos. ¿Se cuenta con las condiciones para administración y monitorización de los posibles efectos adversos que puedan presentar la utilización de ellos (algunos pueden ser letales si no se detectan a tiempo efectos adversos, sumándose el delgado límite de lo terapéutico y tóxico) en niños? En otras palabras, ¿Las condiciones institucionales en que se encuentran actualmente los NNA dependientes del Sename, y que presentan problemas de salud mental, posibilita pensar que los tratamientos psicosociales especializados sean utilizados en primera línea por sobre los tratamientos farmacológicos?

La organización de los cuidados de salud institucionalizados especialmente en Latinoamérica gira alrededor de sistemas políticos y sociales complejos, que muchas veces dejan de lado la verdadera razón de ser y existencia de ellas. Hasta hace poco giraba en un modelo centrado en la acción del médico y la medicina, disciplina dominante y hegemónica en la organización de la salud de la región, centrándose en la patología más que en la persona, dejando fuera el entorno biopsicosocial. En esa línea, las personas deben ser vistas como un ser integral, biopsicosocial y holística que requiere de cuidados culturalmente competentes. Estos saberes disciplinares han sido ampliamente trabajados desde la enfermería, centrados en que la persona es un protagonista activo del proceso de su cuidado e inmerso en un contexto (Rebolledo D., 2013). Precisamente Collado (2016) refiere que existe un control social sobre el cuerpo de las personas, indicándose qué deben hacer, cómo cuidarse y mantener una población productiva, relacionando la construcción profesional con el biopoder, desde una mirada de Foucault.

Por ello, desde la Enfermería se requieren nuevas concepciones que puedan orientar cuidados atingentes a la realidad y necesidades de este grupo, como son los planteamientos de Kérouack citada por Collado \& Castillo (2016), que define los cuidados: "un servicio a la humanidad basado en la observación y la experiencia, que consiste en poner a la persona 
enferma o sana en las mejores condiciones, a fin de que la naturaleza pueda restablecer o recuperar la salud”. Según Collado, ser consciente del poder que constantemente se ejerce sobre las personas que son cuidadas, permitiría cambiar el paradigma vertical a los valores arraigados intrínsecamente en la deontología profesional como son el respeto, confiabilidad, entre otros, buscando la comprensión íntima de los procesos que pasa la persona a lo largo de su vida, permitiendo a su vez, decisiones autónomas como la de consumir un medicamento.

La ética del cuidado promovida por Carol Gilligan citada por Lax A. (2013), refiere que existe, a través de la enfermería, una mayor disposición a escuchar y comprender a las personas:

"dialogando en una relación de intercambio afectivo donde se comparte una determinada visión del mundo y valores. Este carácter subjetivo, empático y narrativo del proceso de enfermedad permite a la persona tanto conocer distintas opciones en su tratamiento más allá de sus convicciones iniciales como de establecer una relación de proximidad moral con la persona que le cuida (...)”.

Sin embargo, la ética del cuidado, no es tanto una manera o un protocolo de cómo ejercer el cuidado, sino un modo o una actitud para llevarlo a cabo, aludiendo a las críticas planteadas a esta perspectiva, pues muchos autores la consideran como difícil de llevarla a la práctica clínica (García L., 2015). Por lo tanto, es necesario definir intervenciones psicosociales protocolizadas y especializadas para el abordaje de las problemáticas de salud mental de estos NNA, junto con limitar y otorgar cuidados profesionales al establecer tratamientos psicofarmacológicos, los cuales deben estar subordinados a las normativas médico-legales que les rigen y considerando la autonomía y toma de decisiones de los mismos. Sin el reguardo explicitado, no debiese administrarse medicamentos, pese a los posibles riesgos que estén implicados en esta decisión; como recaídas, conductas disruptivas, o situaciones que sean de urgencia psiquiátrica.

\section{CONCLUSIONES}

Si bien, las intervenciones psicofarmacológicas en momentos agudos de la enfermedad otorgan beneficios terapéuticos a las personas, las intervenciones psicosociales han demostrado mayor calidad de vida de las personas con problemas de salud mental, disminuyendo el uso de psicofármacos (dosis o uso en tiempo, recaídas, entre otros). Desde la enfermería, es posible establecer las necesidades de salud de manera integral, de cada NNA de la institución para lograr definir las prioridades y lineamientos de acción, contenido en un plan a corto, mediano y largo plazo; a través de una atención continua y oportuna. Es importante destacar que cada NNA, tienen necesidades en salud mental, ya sea por su situación de vulneración de derechos o por los trastornos disruptivos que los llevan a situaciones judiciales.

Se debe considerar el fomento de la toma de decisiones informadas y consensuadas con los NNA, en un trabajo intersectorial y multidisciplinario. A su vez, es importante alertar sobre los peligros asociados a prácticas de salud que no cuenten con una adecuada supervisión o monitorización sobre tratamientos que puedan ser nocivos en la salud de la población descrita. Por ello, se debe convocar a acciones que aseguren un cuidado de calidad en NNA, situación que demanda urgencia de una mejora en el contexto de la crisis institucional del Sistema de Protección a la Infancia y Adolescencia. Los enfermeros y enfermeras deben cautelar que la administración de medicamentos sea segura y otorgar cuidados especializados a quienes lo requieren.

\section{BIBLIOGRAFÍA}

\section{CEPAL. (2014) Panorama Social de Latinoamérica CEPAL. Recuperado de http://www.cepal.org/es/publicaciones/37626 -panorama-social-de-america-latina-2014}

Departamento de Estadísticas en Información en Salud (2015) Indicadores básicos en Salud.Santiago de Chile: DEIS. Recuperado de http://www.deis.cl/

Ministerio de Salud (2013), Programa Nacional de Salud de la Infancia con Enfoque Integral. 
Santiago de Chile: Ministerio de Salud. Recuperado

de

http://web.minsal.cl/sites/default/files/files/2013

_Programa\%20Nacional\%20de\%20Salud\%20de

\%20la\%20infancia\%20con\%20enfoque\%20inte

gral.pdf

Ministerio de Salud (2012) Programa Nacional

Salud Integral Adolescentes. Santiago de Chile:

Miniserio de Salud. Recuperado de http://diprece.minsal.cl/programas-de-

salud/programas-ciclo-vital/informacion-al-

profesional-salud-joven-y-adolescente/

Ministerio de Salud (2000) Plan Nacional de Salud Mental. Santiago de Chile: Miniserio de Salud.Recuperado

de

http://www.minsal.cl/salud-mental

Servicio Nacional de Menores (2015). Anuario Estadístico 2015. Santiago de Chile: SNM. Recuperado

de

http://www.sename.cl/web/anuarios-estadisticossename/

Collado, C.C.S. (2017). Reflexiones sobre enfermería desde la biopolítica: relaciones de poder y cuidado. Cultura de los Cuidados (47), 22-27. Recuperado

de

https://doi.org/10.14198/cuid.2017.47.03

Garcia Moyano, L. (2015). La ética del cuidado y su aplicación en la profesión enfermera. Acta Bioethica, 21(2), 311-317.

Lara-Muñoz, Ma .C., Robles-García, R., Orozco, R., Saltijeral Méndez, Mª.T., Medina-Mora, $M^{\text {a }}$.E., \& Chisholm, D. (2010). Estudio de costoefectividad del tratamiento de la esquizofrenia en México. Salud mental, 33(3), 211-218.

Lax, A. M. (2013). La ética de la vulnerabilidad de Corine Pelluchon The Ethics of Vulnerability by Corine Pelluchon. Revista Internacional de Filosofía, 58, 171-178.

Montenegro, H. (2000). La Psiquiatría del Niño y del Adolescente. Revista chilena de neuropsiquiatría, 38(3), 143-146.

Marmot, M., \& Bell, R. (2012). Fair society, healthy lives. Public Health, 126, S4-S10.

Moreno A. (2013). La ética de la Vulnerabilidad de Corine Pelluchon., Revista internacional de Filosofía, 58, 171 - 178.

Organización para la Cooperación y Desarrollo Económico (2014). Estadísticas de la OCDE sobre la salud. Chile en comparación. Santiago de Chile: OCDE. Recuperado de http://www.oecd.org/els/healthsystems/Briefing-Note-CHILE-2014-inSpanish.pdf
OMS (2013). Plan de Acción sobre Salud Mental 2013-2020.Santiago de Chile: Organización Mundial para la Salud. Recuperado de http://apps.who.int/iris/bitstream/10665/97488/1/ 9789243506029_spa.pdf

Pérez C. (2015). Antipsiquiatras Clasicos y Actuales. Santiago de Chile: Creative Commons (CC BY-NC-ND). Recuperado de https://www.cperezs.org/?p=236

Rebolledo Malpica, D. (2013). Cultura de los Cuidados y las Instituciones de Salud en Nuestros Tiempos en Latinoamérica. Cultura de Los Cuidados, 37, 9-12. Retrieved from http://www.index-f.com/cultura/37pdf/37009.pdf

Rioseco, S., Benajamín, V., Saldivia, S., Cova, S., Melipillán, F., \& Rubi, G, P. (2009). Prevalencia de trastornos psiquiátricos en adolescentes infractores de ley: Estudio casocontrol. Revista chilena de neuro-psiquiatría, 47(3), 190-200.

Benjamín, V., Saldivia, S., \& Pihán, R. (2016). Prevalencias y brechas de hoy; salud mental mañana Acta bioethica, 22(1), 5161. https://dx.doi.org/10.4067/S1726-

\section{X2016000100006}

Benjamín, V., Saldivia, S., de la Barra, F., Melipillán, R., Valdivia, M., \& Kohn, R. (2012). Salud mental infanto-juvenil en Chile y brechas de atención sanitarias. Revista médica de Chile, 140(4), 447-457.

Whitaker, L. (2015). Resistances to critical thinking about brain shrinking 19552015. Ethical Human Psychology and Psychiatry, 17(2), 86-99. Retrieved from http://ezproxy.puc.cl/docview/1727434549?acco $\underline{\text { untid }=16788}$

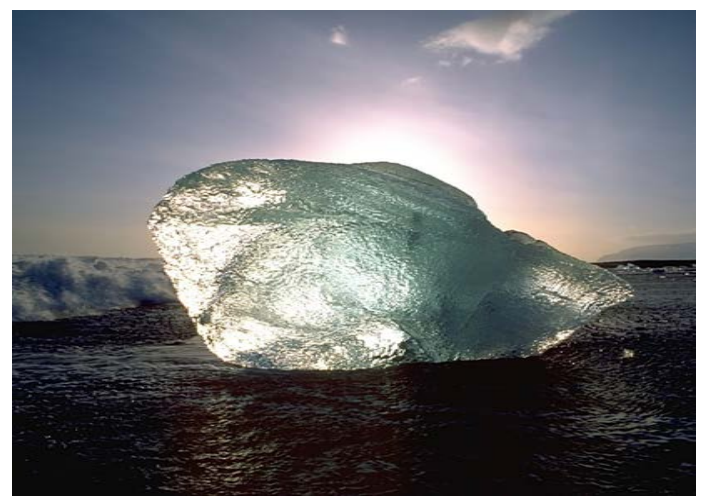

Fuente:Jökulsárlón,s photo. From Wikimedia Commons, the free media repository 